\title{
Multisensor Vehicle Tracking with the Probability Hypothesis Density Filter
}

\author{
Mirko Maehlisch ${ }^{1}$, Roland Schweiger ${ }^{1}$, Werner Ritter ${ }^{2}$ and Klaus Dietmayer ${ }^{1}$ \\ ${ }^{1}$ University of Ulm, Dept. of Measurement, Control and Microtechnology, Ulm, Germany \\ ${ }^{2}$ DaimlerChrysler AG, Research and Technology, Dept. REI/AI, Ulm, Germany \\ uni-ulm.maehlisch@daimlerchrysler.com
}

\begin{abstract}
In this contribution we apply the probability hypothesis density (PHD) filter algorithm for joint tracking of an unknown varying number of targets to automotive environment sensing systems. We use data from a vision and a lidar sensor as well as the vehicle ESP system. After deriving a method to parametrise the algorithm systematically from detection performance statistics we proof the applicability of the method for automotive tracking based on real sensor data.
\end{abstract}

Keywords: Joint Target Tracking, Probability Hypothesis Density, Vehicle Detection, Future Driver Assistance Systems

\section{Introduction}

Several driver assistance systems observing the vehicle environment have already been introduced to the market. The first generation of such systems, introduced for Active Cruise Control (ACC) applications, were realized with long range radar or lidar technology. As a classification feature to distinguish between real targets of interest and background measurements, these systems exclusively used the measured and tracked target velocities over ground. Needless to say, that such a scheme recognizes only moving targets and advanced applications as emergency braking and ACC stop-andgo rely on a robust non-moving object detection, especially in traffic jam situations for instance. In addition, the same is the case for safety systems related to vulnerable road users (VRU), who are often just standing in urban areas. This is why the currently available second generation of ACC systems, solves the problem by taking additional sensor data features into account for a non moving object detection. But due to legal reasons and a remaining uncertainty the fully automatic breaking action is released by the driver after a warning signal.

A further system improvement is expected particularly from heterogeneous sensor data fusion strategies. Future cars will be equipped with additional sensors anyway, such as video sensors for Nightvision and Lane Keeping applications and precise digital maps for navigation purposes. In the best case, using this additional sensor information allows fully automatic decision making without driver interaction, but at least, the systems will produce a faster and more precise response, in matters of identity classification as well as state estimation, because more independent information is available in shorter time.

The most feasible ways to combine data from current automotive sensors are object-list-level or track-totrack fusion approaches allowing the seamless integration of different sensor systems for stand alone applications from possibly different manufacturers with low communication load. But their main drawback is the fact, that although we can achieve good target position and velocity estimation refinements, the improvement related to detection performance, as well as identity or class membership estimation is poor, because each sensors decision making relies only on its individual measurements without taking the other sensors into account. In contrast, low-level or feature-level sensor fusion requires a high bandwith realtime communication network and the integration of raw data interfaces into the sensors. But this effort pays off because all sensor information can be joined before decision making allowing the beneficial use of all available sensor data for centralized detection and classification purposes.

As a step towards such a system we examine the PHD filter as a method for simultaneously managing existence evidence for an unknown varying number of targets over time by incorporating multiple low level sensor measurements without a data association strategy.

\subsection{Related Work}

The PHD filter algorithm introduced by R.Mahler [1], [2], [3] was already implemented for different applications [4], [5] and for Track-before-Detect [6]. To our knowledge there are no applications in the automotive field so far. Instead Kalman filter approaches dominate the area of vehicle tracking in various variants [7], [8].

\subsection{Paper Structure}

After the introduction the paper continues with our sensor setup and shortly describes the sensor data preprocessing in section two. Section three reviews the basics of PHD filtering, presents our sensor and process modeling and introduces modifications to the default algorithm. Afterwards the implementation details of the particle filter are discussed in section four and the 
results are presented in section five. A conclusion with a suggestion of further examinations closes the paper.

\section{Sensors and Data Processing}

Our test vehicle is equipped with a 16-channel multibeam lidar with a distance range of up to 200 meters, a distance resolution of $0.1 \mathrm{~m}$ and horizontal directional resolution of $1^{\circ}$. Additionally we mounted an automotive CMOS camera behind the windshield with standard VGA resolution (see Figure 1). The sensors
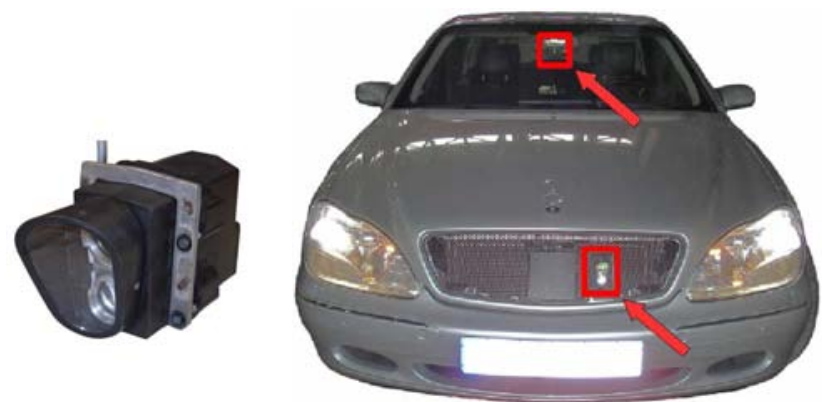

Figure 1: Multibeam lidar sensor (left) and video/lidar integration points (right).

measurements arrive asynchronously at $16 \mathrm{~Hz}$ from the lidar, $25 \mathrm{~Hz}$ from the camera and $50 \mathrm{~Hz}$ from the ESPsystem, but we obtain the measurement timestamps from a central realtime clock. Calculating the spatial alignments of the sensors mounted at different positions on the vehicle enables us to join their measurements in a common fusion reference coordinate system. For example the spatial alignment of the vision and the lidar sensor, together with the intrinsic camera parameters, allow the projection of lidar measurements into the image domain, depicted as diamonds in figure 2 . Like many other systems, we use these projections as an attention control for an image subwindow classifier, the popular Adaboost classifier introduced by Viola and Jones [9], to distinguish between vehicles and background (cyan boxes in fig. 2). The next step is a cluster algorithm, that groups adjacent boxes and their associated lidar echoes. These clusters form the measurement set Z. The ESP measurements consist of the four wheel revolutions and the yawrate of the sensor platform, referred to as the ego vehicle from now, and are obtained from the vehicle CAN-bus.

\section{Data Filtering}

In theroy, the state space of automotive vehicle tracking is a tuple of a random finite set $\Gamma$ [1], [3], representing the multitarget states of the other vehicles and the vector $X_{\text {ego }}$ containing the dynamics of the ego vehicle.

$$
\mathbb{X}=\left(\Gamma, X_{\text {ego }}\right)
$$

Because it is extremely difficult to track within this state space and because the target and the ego-motion can be assumed independent, we decided to filter the two components independently.



Figure 2: Top: geometric sensor models in 3D. Bottom: In the vehicle detection process the lidar echoes (right) define the ROI of the image classifier (left).

\subsection{Ego-Motion Estimation}

The ego-motion of the vehicle is estimated using an Extended Kalman-Filter (EKF) with a state space consisting of the ego-velocity, the ego-yawrate and their accelerations, related to the local vehicle coordinate system, that originates in the center of the rear axis (see fig. 3):

$$
X_{e g o}=\left(v_{e}, \dot{v}_{e}, \dot{\varphi}_{e}, \ddot{\varphi}_{e}\right)^{\prime}
$$

The EKF prediction uses a discrete wiener process acceleration model with the process noise covariance:

$$
Q_{e}=\left(\begin{array}{cc}
G \sigma_{\dot{v}}^{2} G^{\prime} & 0 \\
0 & G \sigma_{\ddot{\varphi}}^{2} G^{\prime}
\end{array}\right), \quad G=(\Delta t, 1)^{\prime}
$$

The ego-motion measurement vector consists of the four wheel revolutions and the yawrate measurement, obtained from the ESP sensors:

$$
Z_{\text {ego }}=\left(R_{F L}, R_{F R}, R_{R L}, R_{R R}, \omega\right)^{\prime}
$$

Assuming Ackermann steering geometry (fig. 3), these measurements are related to the ego-motion state vec- 




Figure 3: Ackermann steering geometry

tor via the ego-motion measurement equations

$$
\begin{aligned}
R_{F L} & =\frac{\sqrt{4 v_{e}^{2}-4 l_{w} v_{e} \dot{\varphi}_{e}+l_{w}^{2} \dot{\varphi}_{e}^{2}+4 l_{l}^{2} \dot{\varphi}_{e}^{2}}}{2 u} \\
R_{F R} & =\frac{\sqrt{4 v_{e}^{2}+4 l_{w} v_{e} \dot{\varphi}_{e}+l_{w}^{2} \dot{\varphi}_{e}^{2}+4 l_{l}^{2} \dot{\varphi}_{e}^{2}}}{2 u} \\
R_{R L} & =\frac{2 v_{e}-l_{w} \dot{\varphi}_{e}}{2 u} \\
R_{R R} & =\frac{2 v_{e}+l_{w} \dot{\varphi}_{e}}{2 u} \\
\omega & =\frac{180}{\pi} \dot{\varphi}_{e}
\end{aligned}
$$

where $\mathrm{u}$ is the dynamic tire circumference.

\subsection{PHD Multitarget Filtering}

The PHD $D(x)$ of a stochastic multitarget process is the first-order statistical moment of the random finite set $\Gamma$, consisting of the individual target states:

$$
D(x)=\sum_{n=0}^{\infty} \frac{1}{n !} \int p\left(\left\{x, y_{1}, \ldots, y_{n}\right\}\right) d y_{1} \ldots d y_{n}
$$

An important property of the PHD is that it integrates to the expected number of targets within the state space:

$$
E(|\Gamma|)=\int D(x) d x
$$

For efficiency reasons, in multitarget filtering tasks the PHD is propagated in a Bayesian-like manner instead of the full joint multitarget probability distribution $p$, because evaluating the latter would introduce an enormous combinatorial complexity.

\subsection{PHD Prediction}

The PHD can be predicted forward in time by:

$$
\begin{aligned}
& D_{k \mid k-1}\left(x_{k} \mid Z_{k-1}\right)=B_{k \mid k-1}\left(x_{k}\right) \\
& +\int p_{\text {death }} f_{k \mid k-1}\left(x_{k} \mid x_{k-1}\right) D_{k-1 \mid k-1}\left(x_{k-1} \mid Z_{k-1}\right)
\end{aligned}
$$

where $B_{k \mid k-1}$ is the PHD of new born targets and $f_{k \mid k-1}$ is the single target Markov transition probability distribution function. Apparently, a mechanism of target spawning can be omitted in the application of automotive vehicle tracking and the Markov transition pdf is implicitly given by the stochastic difference equation:

$$
\begin{aligned}
x_{k} & =f\left(\triangle t, x_{k-1}, c_{k-1}, v_{k-1}\right) \\
& =E\left(c_{k-1}, \triangle t\right) T(\triangle t) x_{k-1}+v_{k-1}
\end{aligned}
$$

where the state vector $x_{k}=(x, \dot{x}, y, \dot{y}, 1)^{\prime}$ of the individual targets was chosen according to the free motion model in homogeneous coordinates. The position coordinates are relative to the ego-vehicle, but the velocities are measured over ground. The vector $c_{k-1}=\left(v_{e}, \dot{\varphi}_{e}\right)^{\prime}$ is the control vector consisting of the ego-motion EKF estimate, predicted to the current lidar or video measurement timestamp, and the process noise is $v_{k-1} \sim \mathcal{N}\left(0, Q_{t}\right)$. The state transition equation combines the individual target motion $\mathrm{T}$ with the ego-motion correction E:

$$
T(\triangle t)=\left(\begin{array}{ccccc}
1 & \triangle t & 0 & 0 & 0 \\
0 & 1 & 0 & 0 & 0 \\
0 & 0 & 1 & \triangle t & 0 \\
0 & 0 & 0 & 1 & 0 \\
0 & 0 & 0 & 0 & 1
\end{array}\right)
$$

$$
E\left(v_{e}, \dot{\varphi}_{e}, \Delta t\right)=\left(\begin{array}{ccccc}
r_{c} & 0 & r_{s} & 0 & t_{x} \\
0 & r_{c} & 0 & r_{s} & 0 \\
-r_{s} & 0 & r_{c} & 0 & t_{y} \\
0 & -r_{s} & 0 & r_{c} & 0 \\
0 & 0 & 0 & 0 & 1
\end{array}\right)
$$

with

$$
\begin{aligned}
r_{s} & =\sin \left(\dot{\varphi}_{e} \triangle t\right) \\
r_{c} & =\cos \left(\dot{\varphi}_{e} \triangle t\right) \\
\triangle x_{e} & =v_{e} \dot{\varphi}_{e}^{-1} \sin \left(\dot{\varphi}_{e} \triangle t\right) \\
\triangle y_{e} & =v_{e} \dot{\varphi}_{e}^{-1}\left(1-\cos \left(\dot{\varphi}_{e} \triangle t\right)\right) \\
t_{x} & =-\triangle y_{e} \sin \left(\dot{\varphi}_{e} \triangle t\right)-\triangle x_{e} \cos \left(\dot{\varphi}_{e} \triangle t\right) \\
t_{y} & =-\triangle y_{e} \cos \left(\dot{\varphi}_{e} \triangle t\right)+\triangle x_{e} \sin \left(\dot{\varphi}_{e} \triangle t\right)
\end{aligned}
$$

The process noise was chosen according to the discrete white noise acceleration model:

$$
Q_{t}=\left(\begin{array}{cc}
G_{t} \sigma_{\ddot{x}}^{2} G_{t}^{\prime} & 0 \\
0 & G_{t} \sigma_{\ddot{y}}^{2} G_{t}^{\prime}
\end{array}\right), \quad G_{t}=\left(\frac{1}{2} \Delta t^{2}, \Delta t\right)^{\prime}
$$

\subsection{PHD Measurement Update}

Given the a-priori PHD $D_{k \mid k-1}$, the measurement set $Z_{k}$ and the individual measurement likelihood function 
$f(z \mid x)$ the correct-step of the PHD recursion is:

$$
\begin{aligned}
& D_{k \mid k}\left(x_{k} \mid Z_{k}\right)=\left(1-p_{s}\right) D_{k \mid k-1}\left(x_{k} \mid Z_{k-1}\right)+ \\
& \sum_{z \in Z_{k}} \frac{p_{s} f\left(z \mid x_{k}\right) D_{k \mid k-1}\left(x_{k} \mid Z_{k-1}\right)}{\lambda c_{k}(z)+p_{s} \int f(z \mid y) D_{k \mid k-1}\left(y \mid Z_{k-1}\right) d y}
\end{aligned}
$$

The parameter $p_{s}$ is the detector sensitivity, thus the probability of detection and $\lambda$ is the average number of false alarms spatially distributed according to $c_{k}$. Since our sensors measure asynchronously, the measurement set $Z_{k}$ at time $k$ either is a set of lidar echoes or each single measurement $z \in Z_{k}$ is a cluster of several image detection boxes. We only classify targets when video measurements arrive, but utilize features from both sensors. Because we use attention control for the video classifier, each image detection cluster has an association to an ROI generating echo of the latest preceding lidar scan. Two scalar features are extracted for classification - the size of the detection clusters from the video classifier and the amplitude of the associated ROI generating lidar echo. Statistics of the feature distributions (see fig. 4) computed from an preclassified evaluation data set, allow the approximation of the existence probabilities of the target candidates conditioned on their individual feature values $p\left(\operatorname{car} \mid f_{a} \wedge f_{s}\right)[10]$. Thresholding the existence probability leads to a binary decision between target or clutter. By varying the threshold and testing the detector against the test database, we obtain the receiver operating characteristics (ROC) [11] of the detection algorithm, assigning a sensitivity $p_{s}$ (probability of detection) and a precision $p_{p}$ (true-positives to number of alarms ratio) to each threshold value (fig. 5). Parameterizing the PHD-Filter with these statistics is not straight forward, because the precision value as a measure of false alarm probability does not fit in the Poisson process model with occurrence rate $\lambda$ utilized in the PHD equations, whereas the sensitivity can be directly incorporated. To solve the issue, we assume a state space with only one possible target position, hence targets can just be present or not. In this case the spatial imprecision of the sensors and the clutter process vanish to unity and the PHD becomes the estimated scalar number of targets :

$$
f(z \mid x)=c_{k}(z)=1, \quad D_{k \mid k}=E_{k \mid k}(|\Gamma|) .
$$

The PHD update equation simplifies to:

$$
\begin{aligned}
& E_{k \mid k}\left(|\Gamma| \mid Z_{k}\right) \\
& =\left(1-p_{s}\right) E_{k \mid k-1}(|\Gamma|)+\sum_{z \in Z_{k}} \frac{p_{s} E_{k \mid k-1}(|\Gamma|)}{\lambda+p_{s} E_{k \mid k-1}(|\Gamma|)} \\
& =\left(1-p_{s}\right) E_{k \mid k-1}(|\Gamma|)+\sum_{z \in Z_{k}} \frac{E_{k \mid k-1}\left(\left|Z_{T P}\right|\right)}{E_{k \mid k-1}\left(\left|Z_{k}\right|\right)} \\
& =\left(1-p_{s}\right) E_{k \mid k-1}(|\Gamma|)+\left|Z_{k}\right| E_{k \mid k-1}\left(p_{p}\right)
\end{aligned}
$$

where $Z_{T P}$ is the set of true positive measurements. We learn that the PHD filter performs an ad-hoc approximation of the detector precision with the a-priori


Figure 4: Feature value distributions of cascade detector cluster sizes (top) and lidar amplitudes (bottom) for false positives (red) and true positives (green) and resulting object likelihood (blue)

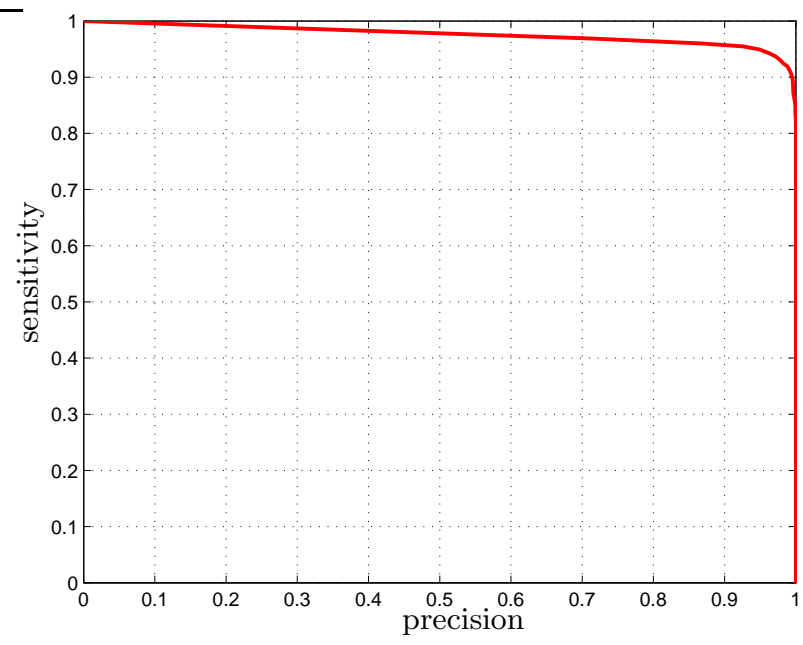

Figure 5: Vehicle detection receiver operating characteristics resulting from the variation of the decision probability threshold 
number of targets estimate by assuming a constant number of false alarms. If we know the precision value a-priori from the ROC, we are able to downweight the spatial a-posteriori state distributions directly with the precision value $p_{p}$. Re-expanding equation (29) for continuous state spaces therefore gives:

$$
\begin{aligned}
& D_{k \mid k}\left(x_{k} \mid Z_{k}\right)=\left(1-p_{s}\right) D_{k \mid k-1}\left(x_{k} \mid Z_{k-1}\right)+ \\
& p_{p} \sum_{z \in Z_{k}} \frac{f(z \mid x) D_{k \mid k-1}\left(x_{k} \mid Z_{k-1}\right)}{\int f(z \mid y) D_{k \mid k-1}\left(y \mid Z_{k-1}\right) d y}
\end{aligned}
$$

In addition, it is possible to discard the threshold operation of our Bayesian two-feature detector because the utilized class membership probabilities are indeed feature-specific - and therefore measurement specific presicion values $p_{p}(z)=p\left(\operatorname{car} \mid f_{a}(z) \wedge f_{s}(z)\right)$. So we abandon target resolving and operate the filter in a track-before-detect mode with:

$$
\begin{array}{r}
D_{k \mid k}\left(x_{k} \mid Z_{k}\right)=\left(1-p_{s}\right) D_{k \mid k-1}\left(x_{k} \mid Z_{k-1}\right)+ \\
\sum_{z \in Z_{k}} \frac{p_{p}(z) f(z \mid x) D_{k \mid k-1}\left(x_{k} \mid Z_{k-1}\right)}{\int f(z \mid y) D_{k \mid k-1}\left(y \mid Z_{k-1}\right) d y}
\end{array}
$$

The probability distribution $f(z \mid x)$ of the lidar sensor model internally consists of the measurement noise, that was determined from empiric examinations and a coordinate transform from the state space, thus the local vehicle coordinate system to the polar lidar measurement coordinate system. For the likelihood of the vision part, we assumed Gaussian statistics by calculating the mean values and the covariances for each detection cluster from the spreading of its boxes in the video image. The likelihood of a position in vehicle coordinates is calculated then by first projecting it into the image domain and evaluating the Gaussian pdf of the measurement at the projected image position.

\subsection{Existence Probability Filtering}

Like [12] we encountered the problem that the PHD shows bad performance in filtering the number of targets. When reviewing equation (27), we learn that besides the first term related to the false negatives, the absolute mass of the a-priori PHD is not used for the final number of targets estimate, but just for computing the expected relation between true positives and over all alarms. In fact, equation (29) shows, how the posterior total PHD weight is nearly completely determined by the cardinality of the measurement set $\left|Z_{k}\right|$ and there is no temporal filtering concerning the number of targets at all. Mahler lately introduced the cardinalized PHD filter (CPHD, [13]) to overcome this problem. Until integrating this new approach into our system, we choose the heuristic approach of filtering the total PHD weight with a constant gain filter after each innovation step. The filtered total weight $E_{k \mid k}^{F}$ is:

$$
E_{k \mid k}^{F}(|\Gamma|)=E_{k \mid k-1}(|\Gamma|)+K\left(E_{k \mid k}(|\Gamma|)-E_{k \mid k-1}(|\Gamma|)\right)
$$

where $E_{k \mid} \cdot(|\Gamma|)$ are the total PHD weights form the original PHD equations and the gain $\mathrm{K}$ is a design parameter. The posterior PHD is reweighted with:

$$
D_{k \mid k}\left(x_{k} \mid Z_{k}\right)=\frac{E_{k \mid k}^{F}(|\Gamma|)}{E_{k \mid k}(|\Gamma|)} D_{k \mid k}\left(x_{k} \mid Z_{k}\right)
$$

\section{Particle Implementation}

The PHD is represented by a weighted set of $\mathrm{N}$ particles $\left(x^{i}, w^{i}\right)$. The sum of the particle weights $w^{i}$ is the expected number of targets within the state space. In our implementation there is no explicit filter initialization, since the birth model accounts for new targets anyway.

\subsection{Prediction}

According to equation (12), the prediction operation consists of the target transition as well as target birth and target death. The birth model is only applied in the video measurement cycles. To approximate the birth PHD, we project each measurement, consisting of a set of video detections with the help of the distance information from the associated ROI-generating lidar echoes back into the local vehicle coordinate system and initialize $N_{b}$ particles with Gaussian noise around these locations. The velocity components are initialized uniformly distributed within $\left[-v_{\max } . . v_{\max }\right]$. The weights of the birth particles are initialized uniformly, too, and sum to the total birth PHD probability mass. The particles of the a-posteriori PHD of the previous time step are propageted through equation (14) by sampling from $v \sim \mathcal{N}\left(0, Q_{t}\right)$. Afterwards, the weights of the propagated particles are multiplied with $1-p_{\text {death }}$ to account for target death.

\subsection{Innovation}

To avoid error-prone clustering operations on the lidar scans, due to their poor spatial resolution (right of fig.2), we chose to treat them as completely unresolved distance profiles, only providing spatial evidence. In contrast the vision sensor allows the extraction of target hypotheses with existence probabilities, utilizing the amplitudes of the ROI generating echoes as described above. This is why there are different innovation procedures, depending on the measurement type. The video update step implements the PHD equations described above. As a first step, we save the prior number of targets estimation $E_{k \mid k-1}(|\Gamma|)=\sum_{i} w_{k \mid k-1}^{i}$. Afterwards, we downweight the whole particle set by multiplying with the false negative probability $\left(1-p_{s}\right)$ to represent the PHD of missed targets. Fore each measurement $z \in Z_{k}$ we thereafter compute the particle likelihoods $f\left(z, x^{i}\right)$ who are first normalized to sum to unity and rescaled with the measurement specific detector presicion $p_{p}(z)$ afterwards. The resulting likelihood vector is added to the particle weights and the process is repeated for the remaining measurements (fig.6/top). The weights now sum to $E_{k \mid k}(|\Gamma|)$ and we are able to compute the posterior number of targets estimate $E_{k \mid k}^{F}(|\Gamma|)$ with equation (32). Finally 


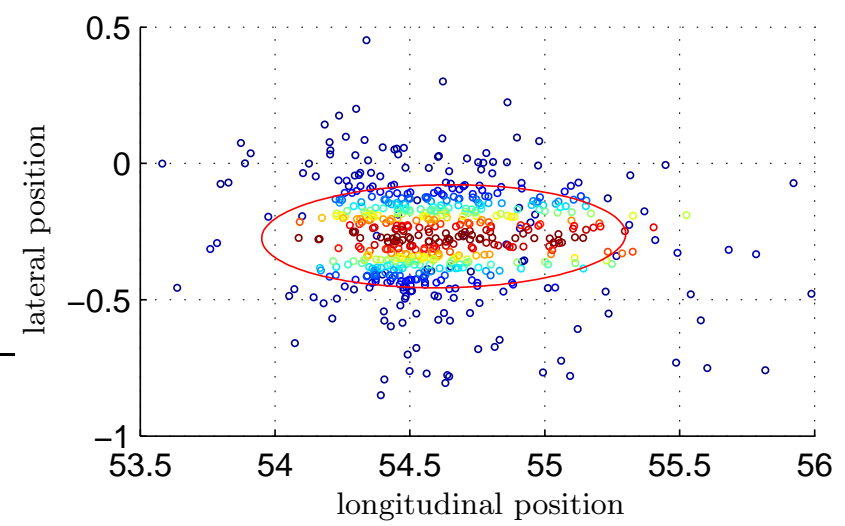

Additionally, the extracted targets from the previous timestep initialize components with the same covariance, but if two components overlap significantly, one of them is pruned. The EM algorithm is now repeated with the remaining $\mathrm{M}$ initial components until no significant change in $\mu_{j}, P_{j}$ or $v_{j}$ occurs between two iterations:

$$
\begin{aligned}
e_{i j} & =\frac{w^{i} v_{j} \mathcal{N}\left(x^{i}, \mu_{j}, P_{j}\right)}{\sum_{l=1}^{M} v_{l} \mathcal{N}\left(x^{i}, \mu_{l}, P_{l}\right)} \\
v_{j} & =\sum_{i=1}^{N} e_{i j} \\
\mu_{j} & =\frac{1}{v_{j}} \sum_{i=1}^{N} e_{i j} x^{i} \\
P_{j} & =\frac{1}{v_{j}} \sum_{i=1}^{N} e_{i j}\left(x^{i}-\mu_{j}\right)\left(x^{i}-\mu_{j}\right)^{\prime}
\end{aligned}
$$

We introduced a pruning step after each iteration, deleting mixture components whose covariance became singular, but the key change is in equation (36), where we omit the re-normalization of the component weights to sum to unity. The new component mixture weights, that sum to the total posterior PHD weight, now have an important meaning. Each mixture weight $v_{j}$ is the PHD mass covered by the j'th component of the mixture and can directly be interpreted as a temporal filtered existence probability of the target hypothesis associated with this mixture component. This is why the unnormalized mixture weights are the natural choice for thresholding to resolve targets after tracking. One single run of the algorithm proved to be sufficient in our examinations.

The lidar innovation step initializes a special temporary lidar particle weight $w_{l}^{i}=0$ for each particle. For each echo we add the sensor likelihood $f\left(z, x^{i}\right)$ to the lidar weights of each particle (fig.6/bottom). Afterwards the lidar weights are normalized to sum to unity, because they only carry state evidence. Actually this is the ordinary SIR filter update.

\subsection{Resampling}

The resampling step preforms importance sampling with the PHD weights as importance density, if an video update preceded or with the lidar weights otherwise. The concept of inversion sampling is well known, so we just note that the total weight of the PHD is preserved in both cases of the resampling step.

\subsection{Target Extraction}

We extract individual targets from the peaks of the PHD surface exclusively after video innovations with a modified version of the EM-algorithm [14] for fitting a Gaussian mixture into weighted data samples $\left(x^{i}, w^{i}\right)$, described in [12]. Like in the birth PHD creation, the detection clusters are projected into vehicle coordinates and gaussian mixture components $\left(\mu_{j}, P_{j}, v_{j}\right)$ are initialized at these locations $\mu_{j}$ with equal predefined covariances $P_{j}$ and equal mixture weights $v_{j}$.

\section{Experiments and Results}

We investigated real data from highway and country road scenes. The system was parameterized with

$$
\begin{aligned}
K & =0.1 \\
p_{\text {death }} & =0.01 \\
\int B_{k \mid k-1} & =0.0126 \\
N_{b} & =50 \\
N & =1000
\end{aligned}
$$

Exemplarily, figure 2 shows a scene with two other vehicles ahead of the sensor platform car and two oncoming vehicles on the left lanes. Figure $7 /$ top shows the total PHD weight for this scene over time for the original PHD $(K=1)$ and the fixed-gain $(K=0.1)$ PHD filters. Obviously the fixed-gain filter introduces the missing memory of the original PHD estimation concerning the number of targets, as it is observable in the low-pass behavior of the total PHD weight. As a result, the number of surviving components of the EM-algorithm is less fluctuating than with the original PHD filter (see figure $7 /$ bottom). We apply the 


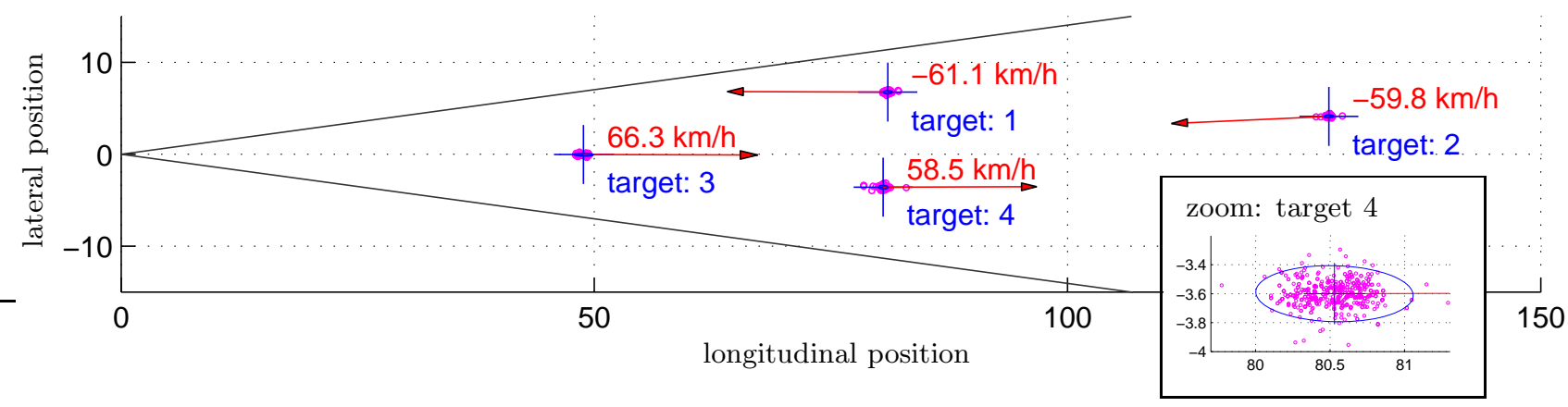

Figure 8: A-posteriori PHD (magenta), projected into a 2D birdview with EM-algorithm estimates of target position (blue crosses), target velocity vectors (arrows) and positional state error covariances (blue 3- $\sigma$ ellipsoids)

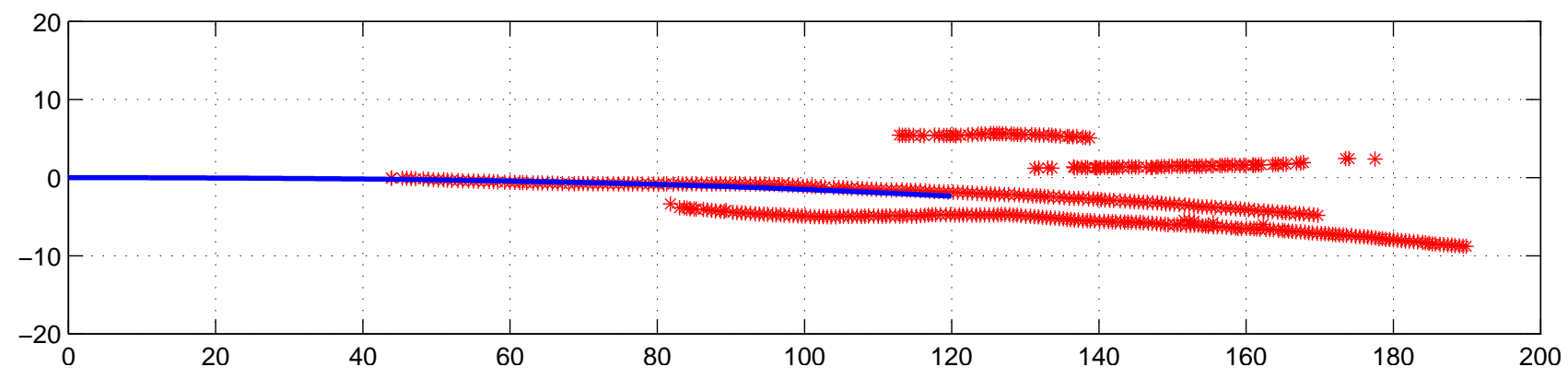

Figure 9: Tracking result: estimated positions of the targets (red asterisks) and the ego vehicle trace (blue line) plotted over time in static local world coordinates
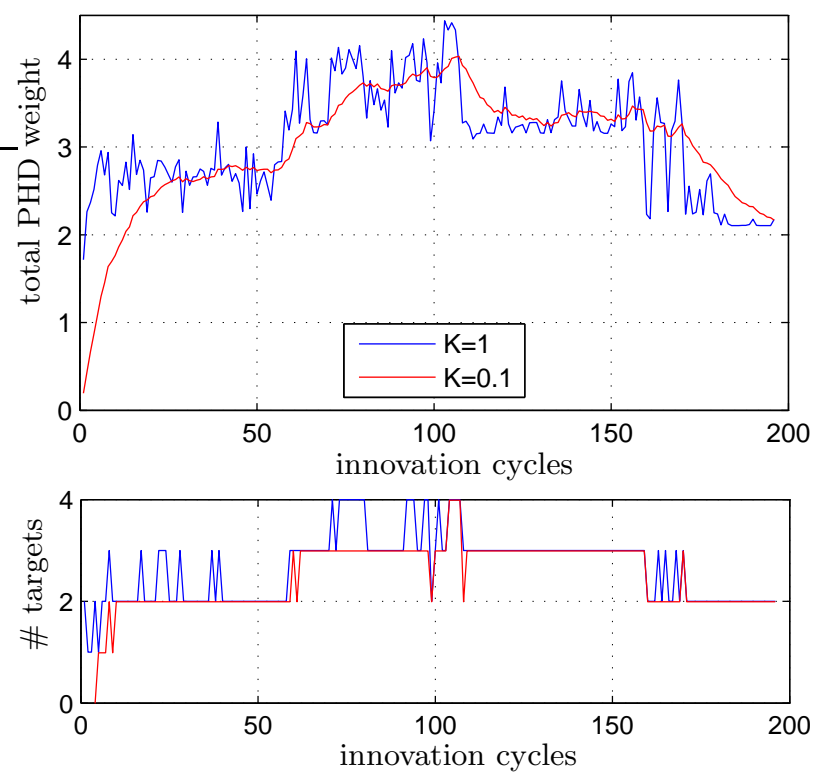

Figure 7: Top: PHD total weight for normal PHD update (red) and fixed-gain filtered version (blue), Bottom: derived number of targets estimate for the two variants over time
EM-algorithm in four dimensions, hence each component has a mean value consisting of position and velocity. Figure 8 shows the posterior PHD at a certain timestep after resampling together with the position and velocity estimates from the EM-algorithm. The whole scene is logged over time in a static world coordinate system, with its origin at the ego vehicles position at time $t=0$ in figure 9 . The red aterisks are the extracted target positions and the blue line is the sensor vehicle trace. Although there are false and missed detections from the video classifier, the PHD filter resolved and tracked up to four targets simultaneously without data association in a stable way.

\section{Conclusion and Further Work}

We developed a detection and tracking system for future driver assistance systems based on the probability hypothesis density filter, that simultaneously omits data association and handles existence evidence of the targets. The novel aspect is the fact, that we incorporate sensor data below the common object list level with the Poisson false alarms distribution model by applying unresolved spatial distance profiles for the lidar sensor and a set of target hypotheses with individual class membership beliefs for the vision sensor instead. In addition the tracker is systematically parameterized from the popular receiver operating characteristics, a common tool in classifier evaluation. Although the results are promising, in the future we have to answer the question, whether the increased computational effort of joint particle filtering pays off for the automotive environment sensing task in comparison to Kalmanfilter approaches with data association and perhaps 
Bayesian existence filtering like the IPDA algorithm.

\section{Acknowledgment}

This research was supported by NIRWARN (NearInfrared Warning), BMBF 01M3157B, Germany.

\section{References}

[1] R. Mahler. Random sets: Unification and computation for information fusion - a retrospective assessment. In Proceedings of International Conference on Information Fusion, pages 1-20, Stockholm, Sweden, 2004.

[2] T.Zajic and R.Mahler. A particle-systems implementation of the phd multitarget tracking filter. In Proceedings of SPIE, Signal Processing, Sensor Fusion, and Target Recognition XII, pages 291299, 2003.

[3] B.Vo, S.Singh, and A.Doucet. Sequential monte carlo methods for multi-target filtering with random finite sets. In IEEE Transactions on Aerospace and Electronic Systems, 2005.

[4] H.Sidenbladh. Multi-target particle filtering for the probability hypothesis density. In Proceedings of International Conference on Information Fusion, Cairns, Australia, 2003.

[5] M. Tobias and A.D. Lanterman. A probability hypothesis density-based multitarget tracker using multiple bistatic range and velocity measurements. In Signal Processing, Sensor Fusion, and Target Recognition XIII, Proc. SPIE 5429, Ed: I. Kadar, 2004.

[6] K.Punithakumar, T.Kirubarajan, and A.Sinha. A sequential monte carlo probability hypothesis density algorithm for multitarget track-before-detect. In SPIE Conference on Signal and Data Processing of Small Targets, San Diego, CA, August 2005.

[7] N.Kaempchen and K.C.J.Dietmayer. IMM vehicle tracking for traffic jam situations on highways. In Proceedings of 7th International Conference on Information Fusion, Stockholm, Sweden, June 2004.

[8] D.Ponsa, A.Lopez, J.Serrat, F.Lumbreras, and T.Graf. Multiple vehicle 3d tracking using an unscented kalman filter. In Proceedings of the 8th International IEEE Conference on Intelligent Transportation Systems, Vienna, Austria, September, 13-16 2005.

[9] P.Viola and M.Jones. Robust real-time object detection. In Second international Workshop on statistical and computational Theories of VisionModeling, Learning, Computing, and Sampling, Vancouver, Canada, July 132001.
[10] M.Maehlisch, R.Schweiger, W.Ritter, and K.C.J.Dietmayer. Sensorfusion using spatiotemporal aligned video and lidar for improved vehicle detection. In Proceedings of IEEE Intelligent Vehicles Symposium, Tokyo, Japan, 2006.

[11] M.Maehlisch, M.Oberländer, O.Löhlein, D.M.Gavrila, and W.Ritter. A multiple detector approach to low-resolution fir pedestrian recognition. In Proceedings of IEEE Intelligent Vehicles Symposium, pages 325-330, Las Vegas, USA, 2005.

[12] O.Erdinc, P.Willett, and Y.Bar-Shalom. Probability hypothesis density filter for multitarget multisensor tracking. In Proceedings of International Conference on Information Fusion, Philadelphia, USA, 2005.

[13] B.T.Vo, B.Vo, and A.Cantoni. The cardinalized probability hypothesis density filter for linear gaussian multi-target models. In Proc. 40th Annual Conf. on Info. Sciences and Systems (CISS'06), Princeton, March 2006.

[14] A.P.Dempster, N.M.Laird, and D.B.Rubin. Maximum-likelihood from incomplete data via the em algorithm. J. Royal Statist. Soc. Ser. B., 39:1-38, 1977. 